\title{
A Comparative Study of Learning Outcomes for Online Learning Platforms
}

\author{
Francois St-Hilaire ${ }^{1}$, Nathan Burns ${ }^{1}$, Robert Belfer ${ }^{1}$, Muhammad Shayan ${ }^{1}$, \\ Ariella Smofsky ${ }^{1}$, Dung Do Vu ${ }^{1}$, Antoine Frau ${ }^{1}$, Joseph Potochny ${ }^{1}$, Farid \\ Faraji $^{1}$, Vincent Pavero ${ }^{1}$, Neroli Ko ${ }^{1}$, Ansona Onyi Ching ${ }^{1}$, Sabina Elkins ${ }^{1}$, \\ Anush Stepanyan ${ }^{1}$, Adela Matajova ${ }^{1}$, Laurent Charlin ${ }^{2}$, Yoshua Bengio ${ }^{2}$, \\ Iulian Vlad Serban ${ }^{1}$, and Ekaterina Kochmar ${ }^{3}$ \\ 1 Korbit Technologies Inc., Canada \\ 2 Quebec Artificial Intelligence Institute (Mila), Canada \\ 3 University of Bath, United Kingdom
}

\begin{abstract}
Personalization and active learning are key aspects to successful learning. These aspects are important to address in intelligent educational applications, as they help systems to adapt and close the gap between students with varying abilities, which becomes increasingly important in the context of online and distance learning. We run a comparative head-to-head study of learning outcomes for two popular online learning platforms: Platform A, which follows a traditional model delivering content over a series of lecture videos and multiple-choice quizzes, and Platform B, which creates a personalized learning environment and provides problem-solving exercises and personalized feedback. We report on the results of our study using pre- and post-assessment quizzes with participants taking courses on an introductory data science topic on two platforms. We observe a statistically significant increase in the learning outcomes on Platform B, highlighting the impact of well-designed and well-engineered technology supporting active learning and problem-based learning in online education. Moreover, the results of the self-assessment questionnaire, where participants reported on perceived learning gains, suggest that participants using Platform B improve their metacognition.
\end{abstract}

Keywords: Online and distance learning - Models of Teaching and Learning · Intelligent and Interactive Technologies · Data Science

\section{Introduction}

We investigate the learning outcomes induced by two popular online learning platforms in a comparative head-to-head study. Platform A is a widely used learning platform that follows a traditional model for online courses: students on this platform learn by watching lecture videos, reading, and testing their knowledge with multiple choice quizzes. In contrast, Platform $\mathrm{A}^{7}$ takes a different

\footnotetext{
${ }^{4} \mathrm{Platform} \mathrm{B}$ is the Korbit learning platform available at www.korbit.ai
} 


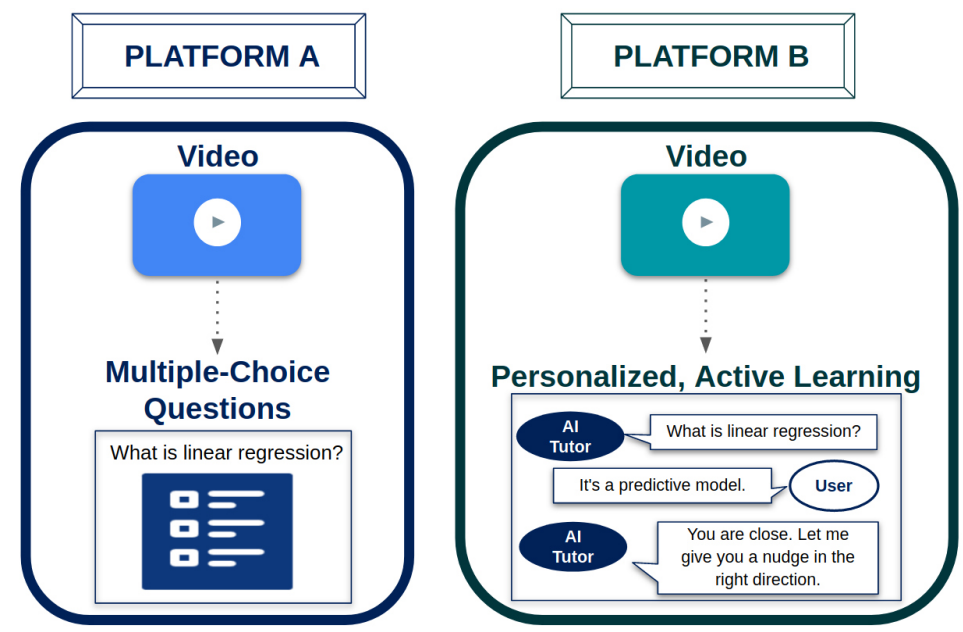

Fig. 1. Platform A follows a traditional learning approach, while Platform B uses a personalized, active learning approach with problem-solving exercises.

approach to online learning, focusing more on active learning and personalization for the students [36. Platform B is powered by an AI tutor, which creates a personalized curriculum for every student and teaches through short lecture videos, interactive problem-solving exercises, mini-projects, and by providing personalized pedagogical interventions mimicking a human tutor. Specifically, Platform B alternates between lecture videos and interactive problem-solving exercises, during which the AI tutor poses questions and shows students problem statements, and students attempt to solve the exercises. Students can also pose their own questions on the material, ask for help, or even skip exercises. The AI tutor addresses each incorrect attempt and each request for help with one of a dozen pedagogical interventions, which are tailored to student's needs, thus ensuring that interactions are personalized. Figure 1 visualizes the differences between the two platforms.

The goal of this study is to measure the efficiency with which students learn on each platform. Since the key difference between the platforms is the fact that Platform B supports personalized, active learning and problem-based learning, our aim is to investigate to what extent such mode of tutoring on online platforms contributes to learning outcomes. We aim to test the following hypothesis:

Hypothesis: Participants who take the course on Platform B have higher learning gains than those who take the course on Platform $A$, because $\mathrm{Platform} \mathrm{B}$ provides a wider and more personalized variety of pedagogical elements to its students. 


\section{Related Work}

Online learning platforms providing a massive number of students with access to learning on various subjects have the potential to revolutionize education [13|18 32|45]. In particular, such platforms have the capability of bridging the gap and addressing inequalities in the society caused by uneven access to in-person teaching 16/41. The current pandemic only exacerbates the need for the high quality online education being accessible to a wide variety of students [14/30.

Nevertheless, the efficacy of online and distance learning has been and continues to be challenged by researchers. It was found that the course design and the mode of teaching strongly influence the way in which students progress [144]. Specifically, it may be hard to address the differences in students' learning needs, styles and aptitudes 915/39/42], and this calls for approaches that can be adapted and personalized to the needs of each particular student. Studies confirm that personalization is key to successful online and distance learning [28|35], such as personalized complexity level and personalized feedback, as it can maximize the learning benefits for each individual student [48].

A number of studies have demonstrated that problem-solving is a highly effective approach for learning in various domains [12 19|20|46|47]. Such problemsolving learning activities can be addressed by intelligent tutoring systems, which are also capable of giving personalized feedback and explanations, incorporating conversational scaffolding, and engaging students into active and problemsolving exercises $[2 / 7 / 8|12| 21|23| 26|27| 29|33| 34]$.

Many studies have been conducted evaluating the impact of educational technology and online learning platforms on student learning outcomes [11/17/24|25] 31 40 42 43. We adopt the well-established pre-post assessment framework, where students are split into intervention groups and their knowledge of the subject is evaluated before and after their assigned intervention.

In contrast to previous studies investigating learning outcomes with intelligent tutoring systems, in this study the AI-powered learning platform, Platform $\mathrm{B}$, is a fully-automated system based on machine learning models [36. The system is trained from scratch on educational content to generate automated, personalized feedback for students and has the ability to automatically generalize to new subjects and improve as it interacts with new students [37/38.

In the context of online and distance learning, students' ability to self-assess, develop self-regulation skills and strategies, plays a crucial role 3[1727. However, many studies show that students generally struggle to evaluate their own knowledge and skills level [5/6/10].

\section{Experimental Setup}

Participants 48 participants completed a 3-hour long course on linear regression using one of the two online platforms. Their learning outcomes were measured before and after the course using pre- and post-assessment quizzes. The 
experiment was run completely online. Participants completing either course were rewarded a $\$ 200$ Amazon gift card.

To recruit participants, we posted ads on social media and sent out emails to student clubs from local universities. Candidates interested in participating had to fill out a questionnaire specifying their field of study, their degree, and whether they have completed courses in machine learning or artificial intelligence.

Candidates were classified as eligible or ineligible based on their answers to this enrollment questionnaire. Specifically, candidates who had or were studying a math-heavy discipline at university (e.g. mathematics, statistics, physics) or who had completed any courses on statistics, machine learning or artificial intelligence were deemed ineligible. As a result, out of the 60 applicants 48 participants were selected. The majority fall into our target audience of undergraduates (89.6\%) studying disciplines not centered around mathematics: health sciences (27.7\%), computer science (23.4\%), cognitive science (12.8\%), among others.

To ensure unbiased setting for the experiments on two platforms, we randomly divided participants into two groups. The first group was asked to study the course on linear regression from Platform A, and the second was asked to study the course on the same subject from Platform B.

Choice of the Material Linear regression was selected as the topic of study on both online platforms since it is one of the most fundamental topics, that is covered early on in any course on machine learning and data science, and the material covering this topic on both platforms is comparable. To yield a fair comparison between the two platforms, extra care was taken to ensure that the linear regression courses were as similar as possible and that the sub-topics covered, the difficulty level, and the length of both courses were carefully aligned.

The linear regression course on Platform B was adapted for this study, combining existing and new content specifically created to align with the sub-topics covered in the Platform A course. As a result, the courses on both platforms contain an introductory session and provide short lecture videos, followed by multiple-choice questions in the case of Platform A and interactive problemsolving exercises in the case of Platform B. The sub-topics taught include numerical variables, correlation, residuals, least squares regression, and evaluation metrics. The course on each platform takes approximately 3 hours to complete.

Study Flow The study ran over a 4-day period with strict deadlines set for the participants. The participants received instructions detailing all the steps they would need to complete on day 1 , and from this point they had 3 days to complete the course. If they completed all necessary steps before the day 3 deadline, they were asked to complete the final post-quiz by the end of day 4 .

All participants were required to take an assessment quiz on linear regression before the course (pre-quiz) and another one after the course (post-quiz). Using pre- and post-quiz scores, we measure learning gains to quantify how efficiently each participant has learned. The pre- and the post-quizzes both consisted of 20 multiple-choice questions and were equally adapted to both courses, meaning 
that any topic or concept mentioned in the quizzes was covered in both courses to an equal extent, ensuring that students mastering the topics using either course would be able to succeed in answering them. In addition, the quizzes went through an independent review process, which ensured that the quiz scores were not inherently biased towards one of the learning platforms. Furthermore, each question of the pre-quiz was isomorphically paired with a question in the postquiz, meaning that the difficulty of the two quizzes was as similar as possible without any questions being identical. This ensured that learning gains were accurately measured without any bias from the differing quiz difficulty $!^{5}$

Safeguarding Against Invalid Results Since the study ran fully online, it was important to take precautionary measures to minimize the chances of the participants cheating or otherwise not following the instructions. We identified the following two potential scenarios: first, a participant might have completed the pre- and the post-quizzes without really studying the course, or going through the course without paying attention, skipping the videos and exercises; second, a participant might have used external resources to find the correct answers on the quizzes. In both cases the participant's scores would be meaningless because it would be completely or almost completely unrelated to the course and the learning platform. To minimize the chances of this happening, we provided participants with very clear instructions on what they were required and what they were not allowed to do, and we required them to upload a completion certificate confirming that they at least went through the whole course on the corresponding platform. We also expect that scheduling the study over 4 days, regardless of how quickly the participants could go through the course, helped discourage them from breezing through the quizzes and the course to immediately get their gift card and ensured there was a delay between their learning and assessment. For Platform B involving problem-solving exercises, we further defined a minimum requirement for participants to attempt at least $80 \%$ of the exercises.

Learning Gains To evaluate which of the two online learning platforms teaches the participants more effectively, we compare Platform A and Platform B based on the average learning gain and normalized learning gain [14] of the participants on each platform. A student's learning gain $g$ is estimated as the difference between their score on the post-quiz and on the pre-quiz as follows:

$$
g=\text { post_score }- \text { pre_score }
$$

where post_score is the student's score on the post-quiz, and pre_score is their score on the pre-quiz. Both scores fall in the interval $[0 \%, 100 \%]$. A student's individual normalized learning gain $g_{\text {norm }}$ is calculated by offsetting a particular student's learning gain against the score range in the ideal scenario in which a student achieves a score of $100 \%$ in the post-quiz:

\footnotetext{
${ }^{5}$ Full content of the assessment quizzes will be made public upon paper acceptance.
} 


$$
g_{n o r m}=\frac{p o s t \_s c o r e-p r e \_s c o r e}{100 \%-\text { pre_score }}
$$

\section{Results and Discussion}

25 participants completed the course on Platform A and 23 participants completed the course on Platform B. One participant on Platform B did not satisfy the requirement of attempting at least $80 \%$ of the exercises and was therefore excluded from the analysis.

It is worth noting that in order to align the courses on the two platforms closely, the personalized curriculum and the programming exercises offered by Platform B were specifically disabled for this study. On the one hand, this allowed us to compare the two platforms on a fair basis and specifically explore the effects of personalized, active learning and problem-based exercises on the learning outcomes in accordance with the formulated hypothesis. On the other hand, given that the personalized curriculum and programming exercises are highlighted on the Platform B website, this may have created a mismatch between students' expectations and their actual learning experience with this platform. Future experiments using the full functionality of Platform B will aim to investigate the effect of other pedagogical elements.

(a)

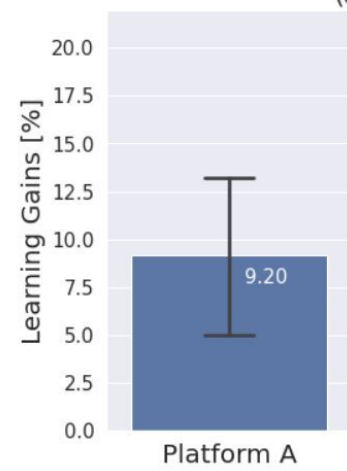

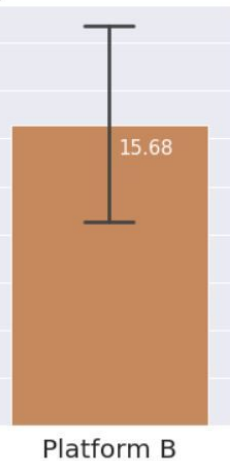

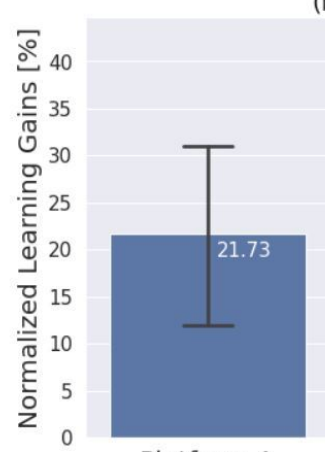

Platform A (b)

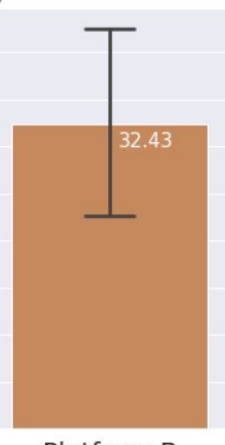

Platform B

Fig. 2. (a) Average learning gains $g$ with $95 \%$ confidence intervals. ${ }^{*}$ (b) Average normalized learning gains $g_{\text {norm }}$ with $95 \%$ confidence intervals. ${ }^{* *}$ Here $^{*}$ and ${ }^{* *}$ indicate a statistically significant difference at $95 \%$ and $90 \%$ confidence level respectively.

Learning Outcomes Average learning gains are shown in Figure 2 for the two learning platforms ${ }^{6}$ The average normalized learning gains $g_{\text {norm }}$ for Platform

\footnotetext{
${ }^{6} 95 \%$ confidence intervals (C.I.) are estimated as: $1.96 * \frac{\text { Standard Deviation }}{\sqrt{\text { Population Size }}}$.
} 
B participants are $49.24 \%$ higher than the average normalized gains for Platform A participants. This difference is statistically significant at the $90 \%$ confidence level $(p=0.068)$. When considering raw learning gains $g$, the Platform B average is $70.43 \%$ higher than the Platform A average with $95 \%$ confidence $(p=0.038)$.

It should be observed that there are 3 participants from Platform A and 2 from Platform B, who showed negative learning gains. These results are hard to interpret because the difficulty level of the pre- and the post-assessment quizzes is the same. A possible explanation for this is that they had little prior knowledge and did not learn the material during the course, but managed to pick the correct answers in the pre-quiz by chance and were less lucky in the post-quiz.

We also observe that the median normalized learning gain $g_{\text {norm }}$ is only slightly higher for Platform B, being at $31.82 \%$ as opposed to $28.57 \%$ for Platform A. With the Platform B average being substantially higher, this could mean that participants who underperform on Platform A are well below the Platform A median, and that participants who overperform on Platform B are well above the Platform B median. This might stem from the fact that Platform B is better adapted to students with different backgrounds and learning needs by having various pedagogical tools and more active learning. However, we believe that more data and further studies would be needed to confirm this assumption.

Further, the medians for raw learning gains $g$ are closer to the respective average values for both platforms: $10 \%$ for Platform A and 15\% for Platform B. This is in line with our hypothesis, but suggests that while the normalization process produces a more meaningful metric for learning gains, it does introduce a lot of variance in the metric due to dividing by the pre-quiz score.

Overall, our hypothesis that learning outcomes are higher for participants on Platform B than participants on Platform A is confirmed by the results presented here, with the average learning gains $g$ and normalized learning gains $g_{\text {norm }}$ being substantially higher for Platform B at $95 \%$ and $90 \%$ statistical significance-level respectively. To explain these results, we theorize that the active learning elements of Platform B play a significant role in the participants' learning experience. The key difference between the two platforms is that Platform B supports active learning and problem-based learning, while Platform A is limited in this regard. To give an idea of the extent to which Platform B provides more active learning than Platform A, we estimated how much time participants spent on different learning activities while completing the course on either platform. Table 1 presents the estimates.

Table 1. Estimated time spent on different learning activities on each platform (minutes and $\%$ of total), excluding time spent on enrollment and assessments

\begin{tabular}{|l|l|l|}
\hline Learning Activity & Platform A & Platform B \\
\hline Watching lecture videos & $46.0 \mathrm{~min} .(33.82 \%)$ & $29.6 \mathrm{~min} .(24.89 \%)$ \\
\hline Reading material & $50.0 \mathrm{~min} .(36.76 \%)$ & $0.0 \mathrm{~min} .(0.00 \%)$ \\
\hline Solving quizzes and exercises & $40.0 \mathrm{~min} .(29.42 \%)$ & $89.3 \mathrm{~min} .(75.11 \%)$ \\
\hline
\end{tabular}


For Platform A, the time estimates for videos and readings come directly from the course website. For the exercises, Platform A gives no time estimate for the 13 multiple choice questions in the course. We estimated it at 40 minutes by testing it ourselves. For Platform B, we have access to the data from the participants' interactions on the platform, which lets us directly calculate estimates for the average time spent watching videos and solving exercises. Based on these estimates, we conclude that participants on Platform B spent more time on active learning than Platform A participants by a factor of 2.23 . We believe that this substantial difference in the active learning time is one of the main reasons for the higher learning gains observed w.r.t. Platform B participants.

We further investigate the participants' behavior on Platform B using the data collected automatically on this platform and report the most insightful correlations observed between participants' behavioral factors and their learning gains. Firstly, we establish a correlation between the total amount of time participants spent on solving exercises and their overall performance on exercises. Specifically, there is a positive correlation between the time spent on exercises and the rate at which participants provided correct answers on the first try $(r=0.34)$. At the same time, the time spent on exercises and the average number of attempts participants needed to get a correct answer are negatively correlated $(r=-0.34)$. This suggests that participants who took more time working on the exercises and formulating their answers performed better. These participants got the correct answer on the first try more often and, on average, took fewer attempts to answer correctly. Secondly, we observe a correlation between the participants' performance on exercises and their learning gains. Specifically, the rate of correct answers on the first try positively correlates with both learning gains $(r=0.44)$ and post-quiz results $(r=0.46)$, and the number of exercises completed positively correlates with the post-quiz score $(r=0.28)$. These correlations may suggest that participants who spent more of their study time on active learning and problem-solving exercises performed better and, as a result, obtained higher post-quiz scores and learning gains. We believe this might indicate the effectiveness of active learning elements on Platform B. One limitation to this hypothesis is that no strong correlation was found between the time spent on exercises and the learning gains or post-quiz scores. We believe this is partly due to the diversity in the background knowledge of the participants, leading to high variance in the pace at which participants completed exercises. More data is needed to thoroughly understand the factors driving the learning gains.

Another interesting observation is that participants who scored higher on the pre-quiz were less receptive to the pedagogical interventions from the AI tutor on Platform B. Specifically, for participants who scored over $50 \%$ on the pre-quiz, there is a stronger negative correlation between their learning gains and the total number of hints they received during the course $(r=-0.58)$. For participants who scored below $50 \%$ on the pre-quiz, this correlation is weaker $(r=-0.32)$. This suggests that the pedagogical interventions were more helpful for the students with lower pre-quiz scores. We believe that the negative correlation between the number of hints received and learning gains is partly explained by the fact 
that pedagogical interventions on Platform B mostly occur when a student is already struggling with an exercise. Therefore participants who received more hints most likely struggled more with the exercises, which, as discussed above, correlates with lower learning gains.

Metacognitive Evaluation In addition to measuring actual learning gains using pre- and post-quizzes, we evaluated various metacognition aspects related to the students' learning experience with the two platforms using a questionnaire where students were asked to report on their experience. Table 2 lists 3 questions we asked the participants on their perceived learning gains.

Table 2. Average perceived learning gains ( $\pm 95 \%$ confidence intervals).

\begin{tabular}{|l|l|c|c|}
\hline ID & Question & Platform A & Platform B \\
\hline Q1 & $\begin{array}{l}\text { How would you rate your comprehension } \\
\text { of the topics you studied? (1-5) }\end{array}$ & $4.16 \pm 0.27$ & $3.65 \pm 0.29$ \\
\hline Q2 & $\begin{array}{l}\text { How well do you think you performed } \\
\text { on the final quiz? (1-100\%) }\end{array}$ & $76.7 \pm 5.41 \%$ & $75.2 \pm 5.63 \%$ \\
\hline Q3 & $\begin{array}{l}\text { How capable would you feel in applying } \\
\text { the skills you learned in a practical setting } \\
\text { (at your job, in a personal project, in your } \\
\text { research, etc.)? (1-5) }\end{array}$ & $3.68 \pm 0.29$ & $3.35 \pm 0.38$ \\
\hline
\end{tabular}

There is a strong correlation between how participants rated their comprehension of the topics studied and their actual learning gains (Q1). This can be seen in Figure 3, which shows the normalized learning gains depending on this subjective rating. The correlation is higher for Platform B participants $(r=0.41)$ than for Platform A participants $(r=0.26)$. This suggests that Platform B gave participants a more accurate understanding of their own knowledge level and helped improve their metacognition. For Q2 and Q3 on perceived learning, no correlation was found with the actual learning gains.

Despite the fact that perceived learning gains are strongly correlated with the actual learning gains for Platform B, on average, participants from Platform B reported lower levels of comprehension in absolute terms (Q1), and the difference from the Platform A average is significant $(p<0.05)$. This directly contradicts the fact that Platform B participants obtained higher learning gains on average. In addition, the results for Q2 and Q3 suggest that the Platform B participants' perception of their performance on the post-quiz and their perceived capability to apply the skills they learned are slightly lower than for Platform A participants. We note that, in line with previous work, this indicates that students generally struggle to evaluate their own knowledge and skills level [56]10]. Furthermore, we hypothesize that the reason for this contradictory result is the presence of some frustrating elements in Platform B: for instance, one common source of confusion reported for Platform B is the fact that the AI tutor did not always 


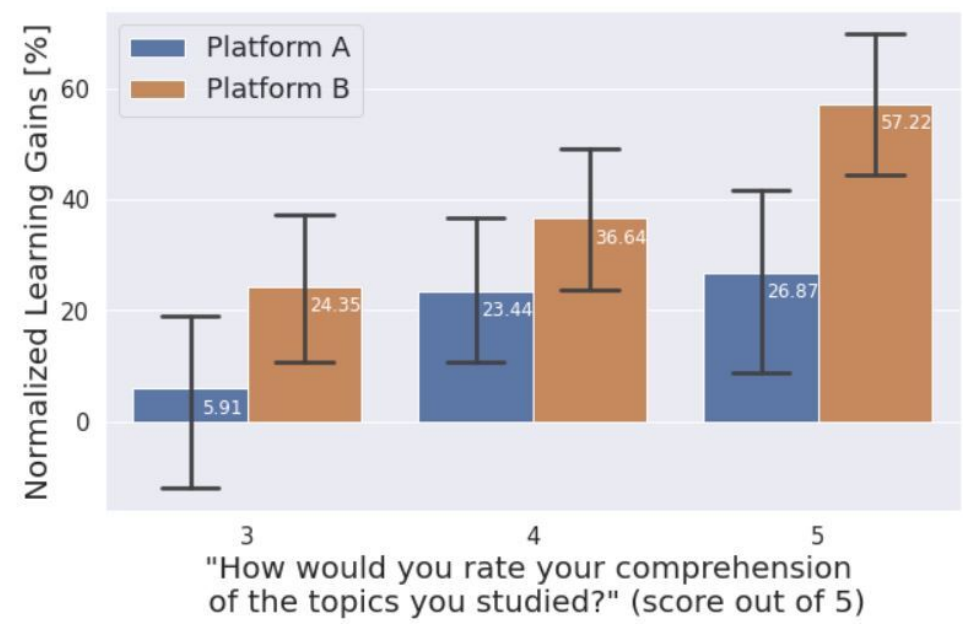

Fig. 3. Normalized learning gains for each self-assessed comprehension rating with $95 \%$ confidence intervals. Only 1 participant gave a score lower than 3 (not shown here).

understand participants' answers. On the one hand, the AI tutor on Platform $B$ engaging in a dialogue with the participants and providing them with the interactive problem-solving exercises strongly contributes to higher learning gains on Platform B. On the other hand, this aspect makes Platform B more technically challenging to implement than the more traditional approach taken by Platform A, and we hypothesize, in line with previous research [22 49], that improvements in the AI tutor's understanding of students' answers and feedback might contribute to higher perceived learning gains in students. Future research should investigate this hypothesis.

\section{Conclusions}

This study compared two popular online learning platforms with respect to the learning outcomes and metacognition induced by the platforms. The first platform, Platform A, is a widely-used learning platform that follows a traditional model for online courses: students on this platform learn by watching lecture videos, reading, and testing their knowledge with multiple choice quizzes. The second platform, Platform B, focuses on active learning and personalization, where each student learns through short lecture videos and interactive problemsolving exercises accompanied by personalized feedback.

We assessed the learning gains of 47 participants after a 3-hour long course on linear regression, with 25 participants taking the course on Platform A and 22 on Platform B. We observed that the average learning gain for Platform B is $70.43 \%$ higher than on Platform A $(p<0.05)$. This supports the hypothesis that participants on Platform B have higher learning gains than those who take the 
course on Platform A, because Platform B provides a wider and more personalized variety of pedagogical elements to its students. Furthermore, the average normalized learning gain for Platform A participants is $21.73 \%$, and $32.43 \%$ for Platform B participants. With Platform B producing $49.24 \%$ higher $(p<0.1)$ learning gains relative to Platform A, the results support the hypothesis that Platform B teaches more effectively. However, more data should be collected to validate the same hypothesis w.r.t. normalized learning gains.

In addition, perceived learning gains and metacognition abilities were assessed with a feedback questionnaire filled out by participants after the postassessment quiz. It was found that Platform A participants report higher perceived learning gains despite the fact that their actual learning gains are lower on average. Higher correlation between perceived learning and actual learning gains for Platform B participants suggests that Platform B induces better metacognition. Lower perceived learning gains on Platform B can be explained by the fact that this platform involves more complex teaching elements. These elements may contribute to higher actual learning gains, they may at the same time also be a source of frustration and confusion for some participants. Future research should look more closely into the factors that affect the perceived learning for participants, and should investigate to what extent the participants benefit from the course being adapted to their learning needs and preferences.

\section{References}

1. Adedoyin, O. B., Soykan, E.: Covid-19 pandemic and online learning: the challenges and opportunities. Interactive Learning Environments, 1-13 (2020)

2. Albacete, P., Jordan, P., Katz, S., Chounta, I.A., McLaren, B.M.: The Impact of Student Model Updates on Contingent Scaffolding in a Natural-Language Tutoring System. In: International Conference on Artificial Intelligence in Education, pp. 37-47. Springer (2019)

3. Barokas, J., Ketterl, M., Brooks, C., Greer, J.: Lecture capture: Student perceptions, expectations, and behaviors. In: J. Sanchez \& K. Zhang (Eds.), World Conference on E-Learning in Corporate, Government, Healthcare, and Higher Educatio, pp. 424-431 (2010)

4. Basilaia, G., Kvavadze, D.: Transition to online education in schools during a SARSCoV-2 coronavirus (COVID-19) pandemic in Georgia. Pedagogical Research, 5(4) (2020)

5. Brown, G. T. L., Harris, L. R.: Student self-assessment. In J. H. McMillan (Ed.). The SAGE handbook of research on classroom assessment (pp. 367-393). Thousand Oaks, CA: Sage (2013)

6. Brown, G. T., Andrade, H. L., Chen, F.: Accuracy in student self-assessment: directions and cautions for research. Assessment in Education: Principles, Policy \& Practice, 22(4), 444-457 (2015)

7. Büdenbender, J. and Frischauf, A. and Goguadze, G. and Melis, E. and Libbrecht, P. and Ullrich, C.: Using computer algebra systems as cognitive tools. International Conference on Intelligent Tutoring Systems, 802-810 (2002)

8. Chi, M., Koedinger, K., Gordon, G., Jordan, P., Vanlehn, K.: Instructional Factors Analysis: A Cognitive Model For Multiple Instructional Interventions. In: EDM 2011 
- Proceedings of the 4th International Conference on Educational Data Mining, pp. 61-70 (2011)

9. Coffield, F. J., Moseley, D. V., Hall, E., Ecclestone, K.: Learning Styles for Post 16 Learners: What Do We Know? London: Learning and Skills Research Centre/University of Newcastle upon Tyne (2004)

10. Crowell, T. L.: Student Self Grading: Perception vs. Reality. American Journal of Educational Research 3(4), 450-455 (2015). https://doi.org/10.12691/education-3$4-10$

11. Demmans Epp, C., Phirangee, K., Hewitt, J., Perfetti, C. A.: Learning management system and course influences on student actions and learning experiences. Educational Technology, Research and Development (ETRD), 68(6), 3263-3297 (2020)

12. Fossati, D., Di Eugenio, B., Ohlsson, S., Brown, C., Chen, L.: Data driven automatic feedback generation in the iList intelligent tutoring system. Technology, Instruction, Cognition and Learning, 10(1), 5-26 (2015)

13. Graesser, A., VanLehn, K., Rose, C., Jordan, P., Harter, D.: Intelligent tutoring systems with conversational dialogue. AI Magazine, 22(4), 39-51 (2001)

14. Hake, R. R.: Interactive-engagement versus traditional methods: A six-thousandstudent survey of mechanics test data for introductory physics courses. American journal of Physics 66(1), 64-74 (1998)

15. Honey, P., Mumford A.: The Manual of Learning Styles. Peter Honey Publications, Maidenhead (1992)

16. Hrastinski, S., Stenbom, S., Benjaminsson, S., Jansson, M.: Identifying and exploring the effects of different types of tutor questions in individual online synchronous tutoring in mathematics. Interactive Learning Environments, 1-13 (2019)

17. Kashihara, A., Hasegawa, S.: A model of meta-learning for web-based navigational learning. International Journal of Advanced Technology for Learning, 2(4), 198-206 (2005)

18. Koedinger, K., Corbett, A.: Cognitive tutors: Technology bringing learning sciences to the classroom. R.K. Sawyer (Ed.), The Cambridge handbook of the learning sciences, Cambridge University Press, New York, NY, pp. 61-78 (2006)

19. Kolb, D. A.: Experiential learning experience as the source of learning and development, New Jersey, Prentice Hall (1984)

20. Kumar, A. N.: Results from the evaluation of the effectiveness of an online tutor on expression evaluation. ACM SIGCSE Bulletin 37(1), 216-220 (2005)

21. Kumar, A.N.: Generation of problems, answers, grade, and feedback - case study of a fully automated tutor. Journal on Educational Resources in Computing (JERIC), $\mathbf{5}(3), 3-$ es (2005)

22. Lehman, B., D'Mello, S., Graesser, A.: Confusion and complex learning during interactions with computer learning environments. The Internet and Higher Education 15(3), 184-194 (2012)

23. Lin, C. F., Yeh, Y. C., Hung, Y. H., Chang, R. I.: Data mining for providing a personalized learning path in creativity: An application of decision trees. Computers \& Education 68, 199-210 (2013). https://doi.org/https://doi.org/10.1016/j.compedu.2013.05.009.

24. Ma, W., Adesope, O. O., Nesbit, J. C., Liu, Q.: Intelligent Tutoring Systems and Learning Outcomes: A Meta-Analysis, Journal of Educational Psychology (2014)

25. Mark, M.A. and Greer, J.E.: Evaluation methodologies for intelligent tutoring systems. Journal of Artificial Intelligence in Education, 4, 129-129 (1993)

26. Melis, E., Siekmann, J.: ActiveMath: An Intelligent Tutoring System for Mathematics. Artificial Intelligence and Soft Computing, 91-101 (2004) 
27. Munshi, A., Biswas, G.: Personalization in OELEs: Developing a Data-Driven Framework to Model and Scaffold SRL Processes. In: International Conference on Artificial Intelligence in Education, pp. 354-358. Springer (2019)

28. Narciss, S., Sosnovsky, S., Schnaubert, L., Andrès, E., Eichelmann, A., Goguadze, G., Melis, E.: Exploring feedback and student characteristics relevant for personalizing feedback strategies. Computers \& Education, 71, 56-76 (2014)

29. Nye, B. D., Graesser, A. C., Hu, X.: AutoTutor and family: A review of 17 years of natural language tutoring. International Journal of Artificial Intelligence in Education, 24(4), 427-469 (2014)

30. Onyema, E. M., Eucheria, N. C., Obafemi, F. A., Sen, S., Atonye, F. G., Sharma, A., Alsayed, A. O.: Impact of Coronavirus pandemic on education. Journal of Education and Practice, 11(13), 108-121 (2020)

31. Penstein, C. R., Moore, J., VanLehn, K., Allbritton, D.: A Comparative Evaluation of Socratic versus Didactic Tutoring. Annual Meeting of the Cognitive Science Society, 23 (2001)

32. Psotka, J., Massey, D., Mutter, S.: Intelligent tutoring systems: Lessons learned. Lawrence Erlbaum Associates, Hillsdale, NJ (1988)

33. Rus, V., Stefanescu, D., Baggett, W., Niraula, N., Franceschetti, D., Graesser, A.C.: Macro-adaptation in conversational intelligent tutoring matters. In: International Conference on Intelligent Tutoring Systems, pp. 242-247. Springer (2014)

34. Rus, V., Stefanescu, D., Niraula, N., Graesser, A.C.: DeepTutor: towards macroand micro-adaptive conversational intelligent tutoring at scale. In: Proceedings of the first ACM conference on Learning@ Scale conference, pp. 209-210 (2014)

35. Sampson, D., Karagiannidis, C.: Personalised learning: educational, technological and standarisation perspective. Digital Education Review, (4), 24-39 (2002)

36. Serban, I. V., Gupta, V., Kochmar, E., Dung, Vu, D. D., Belfer, R., Pineau, J., Courville, A., Charlin, L., Bengio, Y.: A Large-Scale, Open-Domain, Mixed-Interface Dialogue-Based ITS for STEM. In: International Conference on Artificial Intelligence in Education, pp. 387-392. Springer, Cham (2020)

37. Kochmar, E., Do Vu, D., Belfer, R., Gupta, V., Serban, I. V., Pineau, J. Automated Personalized Feedback Improves Learning Gains in An Intelligent Tutoring System. In International Conference on Artificial Intelligence in Education (pp. 140-146). Springer, Cham.

38. Grenander, M., Belfer, R., Kochmar, E., Serban, I. V., St-Hilaire, F., Cheung, J. C. (2021). Deep Discourse Analysis for Generating Personalized Feedback in Intelligent Tutor Systems. In The 11th Symposium on Educational Advances in Artificial Intelligence (2021)

39. Stash, N. V., Cristea, A. I., De Bra, P. M.: Authoring of learning styles in adaptive hypermedia: problems and solutions. In: Proceedings of the 13th international World Wide Web conference on Alternate track papers \& posters, pp. 114-123 (2004)

40. Tan, Y., Quintana, R. M.: What can we learn about learner interaction when one course is hosted on two MOOC platforms? In: Companion Proceedings to the International Conference on Learning Analytics and Knowledge (LAK), pp. 155-156 (2019)

41. Tomkins, S., Ramesh, A., Getoor, L.: Predicting Post-Test Performance from Online Student Behavior: A High School MOOC Case Study. International Educational Data Mining Society (2016)

42. VanLehn, K., Graesser, A.C., Jackson, G., Jordan, P., Olney, A., Rose, C.P.: When are tutorial dialogues more effective than reading? Cognitive Science, 31(1), 3-62 (2007) 
43. VanLehn, K.: The Relative Effectiveness of Human Tutoring, Intelligent Tutoring Systems, and Other Tutoring Systems, Educational Psychologist (2011)

44. Vigentini, L., Clayphan, A.: Pacing through MOOCs: course design or teaching effect? In: EDM, pp. 572-573 (2015)

45. Wang, Y., Paquette, L., Baker, R.: A longitudinal study on learner career advancement in MOOCs. Journal of Learning Analytics, 1(3), 203-206 (2014)

46. Wood, D., Wood, H.: Vygotsky, Tutoring and Learning. Oxford review of Education 22(1), 5-16 (1996)

47. Woolf, B.: Building intelligent interactive tutors Morgan Kaufmann Publishers, Burlington, MA (2009)

48. Yin, B., Patikorn, T., Botelho, A. F., Heffernan, N. T.: Observing personalizations in learning: Identifying heterogeneous treatment effects using causal trees. In Proceedings of the Fourth (2017) ACM Conference on Learning@ Scale, pp. 299-302 (2017)

49. Zhang, Y., Paquette, L., Baker, R. S., Ocumpaugh, J., Bosch, N., Munshi, A., Biswas, G.: The relationship between confusion and metacognitive strategies in Betty's Brain. In: Proceedings of the Tenth International Conference on Learning Analytics \& Knowledge, pp. 276-284 (2020) 